\title{
Evaluation of Peripheral Blood Neutrophil Functions after an Oral Carbohydrate Overload in Obese and Insulin Resistant Horses
}

\section{Constanza Salinas}

Universidad Austral de Chile

\section{Gabriel Espinosa}

Universidad Austral de Chile

\section{Natalia Morales}

Universidad Austral de Chile

Claudio Henriquez

Universidad Austral de Chile

\section{Gabriel Moran}

Universidad Austral de Chile

\section{Gonzalo Gajardo}

Universidad Austral de Chile

\section{Rafael A Burgos}

Universidad Austral de Chile

Benjamin Uberti ( $\sim$ buberti@gmail.com )

Universidad Austral de Chile https://orcid.org/0000-0002-3888-6746

\section{Research article}

Keywords: Horse, obesity, neutrophils, oxidative burst, phagocytosis, apoptosis

Posted Date: October 20th, 2020

DOI: https://doi.org/10.21203/rs.3.rs-90317/v1

License: (1) (1) This work is licensed under a Creative Commons Attribution 4.0 International License. Read Full License 


\section{Abstract}

Background: Obesity and insulin resistance (IR) are conditions of increasing prevalence in populations of equids worldwide. The immune impairment described in metabolic dysfunction status in humans has been extensively reported with minimal data regarding horses. The objective of the study was to evaluate the effect of obesity as an isolated factor and in association with insulin resistance on apoptosis, phagocytosis and oxidative burst activity of neutrophils isolated from peripheral blood of lean and obese adult horses with or without insulin resistance, basally and after induction of hyperglycemia through an oral glucose test.

Results: No differences in apoptosis were observed between experimental groups at any time point. Phagocytic capacity was significantly diminished at baseline in the obese-IR group $(P<0.05)$ but increased after stimulation of hyperglycemia $(P=0.007)$. Basal reactive oxygen species production differed significantly ( $P=0.0001)$ between the obese-insulin sensitive (IS) and lean-IS or obese-IR groups, and decreased significantly after stimulation of hyperglycemia in the lean-IS and obese-IS groups $(\mathrm{P}<0.05)$.

Conclusions: Results from this study showed that both metabolic status itself, and acute hyperglycemia, are factors that influence PMNs functionality in horses, specifically phagocytosis and oxidative burst. This indicates the need for close monitoring of immune function in horses with inflammatory disease and concurrent obesity and insulin resistance.

\section{Background}

Endocrine disorders associated to obesity represent a worldwide health problem, with special importance in humans and companion animals [1, 2]. In horses, the epidemic of obesity is a growing and concerning problem with rates of obesity as high as $51 \%$ reported $[3,4]$. Classically, as in other species, obesity in horses has been considered a risk factor for the development of insulin resistance (IR), which in conjunction with hyperinsulinemia is referred to as insulin dysregulation (ID) in equine populations [5]. Abnormal insulin metabolism is a key feature of endocrinopathic disorders and has been directly linked with the development of the life-threatening condition laminitis [6-9]. The close relation between obesity, IR and immune compromise is well recognized in humans and mice $[10,11]$, while in horses this relation is not fully understood with fewer reports on the topic [12-15]. Although obesity and IR are closely linked, it must be acknowledged that these conditions can develop independently.

Obesity is not only a predictor of progression of later endocrine disease, but an altered metabolic state in itself [16-18]. The adipose tissue is an active endocrine organ, whose dysfunctional activity is mainly characterized by altered secretion of adipokines and increased expression of proinflammatory cytokines (principally tumor necrosis factor alpha (TNFa) and interleukin 1 (IL-1)) [19]. The activation of the immune system as consequence of metabolic dysfunction, leads to a characteristic low-grade chronic inflammation state reported in obese humans [20-22]. In horses, the state of chronic inflammation 
associated to endocrine dysfunction remains unclear, with inconsistent data reports linking body condition score (BCS) and secretion of proinflammatory cytokines [13, 23-25]. The complex interactions between the endocrine and innate immune system are still incompletely understood in equids [26-28].

Neutrophils (polymorphonuclear cells, PMNs) are part of the innate immune system and play a major role in host defense, as the first cellular type to be recruited to fight inflammation and infection [29]. PMNs are the most abundant leukocyte cell type on the circulating blood of mammals, including horses [30]. In humans with poor metabolic control associated to metabolic conditions such as type 2 diabetes mellitus [31,32], high levels of circulating glucose and insulin are able to impair neutrophil functions, with hyperglycemia acting as an important downregulatory factor for PMNs activity [33, 34]. In healthy humans, acute hyperglycemia has been associated with a decrease in neutrophil chemotaxis and phagocytosis [35], impaired reactive oxygen species (ROS) production [36] and inhibition of neutrophils extracellular traps (NETs) formation [37]. Although horses are known as good regulators of blood glucose levels, with pancreatic exhaustion occurring infrequently [19], limited data exists regarding neutrophil function in horses with energetic metabolism abnormalities. This study group recently reported results in obese non-insulin dysregulated horses [38], and only a few studies have reported altered PMNs function in hyperinsulinemic-obese horses [13] and aged horses suffering from pituitary pars intermedia dysfunction [14]. The effect of endocrine disturbances on PMNs functions in horses remains unknown, and more studies are needed to understand the close relation between the immune system and endocrine disorders. To the authors' knowledge, minimal data is available regarding the assessment of neutrophil functionality of horses with metabolic disturbances, and no previous studies have been reported regarding the in vivo effect of acute induced hyperglycemia on equine neutrophil functions. Therefore, the purpose of the study is to evaluate apoptosis, phagocytosis and oxidative burst activity of neutrophils from peripheral blood, basally and after induction of acute hyperglycemia through an oral glucose test, in groups of lean and obese adult horses with or without insulin resistance.

\section{Methods}

\subsection{Animals}

Seventeen adult horses, including eight geldings and nine non-pregnant mares were enrolled in the study. Horses were privately owned or part of the Austral University of Chile veterinary teaching hospital research herd. An informed consent was signed before the performance of the study for privately owned horses. Animals did not participate in any other studies for at least a month prior to enrollment in this study. Breeds included eight Chilean horses and nine crossbreed horses. To ensure animals' optimal health conditions, a complete clinical examination and blood analyses were performed before the onset of the study. Animals were selected and allocated into three groups based on their body condition score (BCS) and metabolic status (presence or absence of insulin resistance). BCS was assessed by two experienced observers, based on a 9-point scale [39]. To allocate horses in their correspondent group, all horses underwent an insulin tolerance test (ITT) as previously described before the performance of the study to determine their tissue insulin sensitivity or insulin resistance status [40]. The control lean insulin 
sensitive group (lean-IS) included seven horses with a regular BCS (4-6/9) and absence of insulin resistance. The second group comprised five obese insulin sensitive (obese-IS) horses with an obese BCS $(\geq 8 / 9)$ and absence of insulin resistance. The third and final group, obese insulin resistant (obese-IR), included five animals with an obese BCS $(\geq 8 / 9)$ and presence of tissue insulin resistance.

Characteristics data from the three groups are summarized in Table 1 . The study was approved by the Bioethics Committee for the Use of Animals in Research of Austral University of Chile (Resolution $\mathrm{N}^{\circ}$ 308/2018). After experimentation, all animals resumed normal previous activities in their herds of origin.

Table 1

Characteristics and endocrine data of the animal groups selected and enrolled in the study, lean-IS, obeseIS and obese-IR.

\begin{tabular}{|llll|}
\hline Measure (units) & Lean-IS & Obese-IS & Obese-IR \\
\hline Gender & $5 \mathrm{M} ; 2 \mathrm{~F}$ & $2 \mathrm{M} ; 3 \mathrm{~F}$ & $1 \mathrm{M} ; 4 \mathrm{~F}$ \\
\hline Age (years) & $12 \pm 5^{\mathrm{a}}$ & $12 \pm 7^{\mathrm{a}}$ & $16 \pm 3^{\mathrm{a}}$ \\
\hline Weight $(\mathrm{kg})$ & $329 \pm 65^{\mathrm{a}}$ & $433 \pm 95^{\mathrm{b}}$ & $488 \pm 22^{\mathrm{b}}$ \\
\hline BCS (1-9) & $6(4-6)$ & $9(8-9)$ & $9(8-9)$ \\
\hline Basal glucose IRT (mg/dL) & $84.8 \pm 4.4^{\mathrm{a}}$ & $92.5 \pm 4.9^{\mathrm{a}}$ & $93 \pm 8.5^{\mathrm{a}}$ \\
\hline Glucose 30 min post-IRT $(\mathrm{mg} / \mathrm{dL})$ & $20.1 \pm 0.3^{\mathrm{a}}$ & $29 \pm 7.0^{\mathrm{a}}$ & $59 \pm 18.4^{\mathrm{b}}$ \\
\hline
\end{tabular}

Data is presented as mean \pm standard deviation (SD). Body condition score data is presented as median (range). Within a row, values with different superscripts differs significantly $(P<0.05)$. Lean-IS $n=7$; obese-IS $n=5$ and obese-IR $n=5$; insulin sensitive; IR, insulin resistance.

\subsection{Study design}

All horses underwent an infeed oral glucose test (infeed-OGT). The night before the test, horses were moved to stalls and fasted overnight. Free access to water was allowed. At 8:00 AM, horses were fed $1.0 \mathrm{~g}$ dextrose $/ \mathrm{kg}, 0.15 \%$ of body weight alfalfa cubes and $200 \mathrm{~g}$ of wheat bran mixed with $200 \mathrm{~mL}$ of water, as previously described [41]. Horses were allowed a 15 min period to eat their meals, after which, if the meal wasn't eaten, the test was discarded and repeated after a wash-out period of two days. Before the meal was offered to the horses, jugular blood samples were taken for the measurement of basal blood glucose, insulin and further laboratory analysis. After the meal was offered, serial blood samples were collected at 30, 60, 90 and 120 min for the analysis of blood glucose. At 120 min, blood samples were taken for the measurement of glucose, insulin and for laboratory analysis. Insulin was determined at baseline (time 0 ) and 120 minutes. Animals with a fasting insulin concentration $>20 \mu \mathrm{lU} / \mathrm{mL}$, or $\geq 85$ $\mu \mathrm{IU} / \mathrm{mL}$ at $120 \mathrm{~min}$ were excluded from the study. Once the test was completed, animals were returned to paddocks. Tests were performed at the Austral University of Chile veterinary teaching hospital, with the exception of five horses that were tested on field, following the same protocol described above.

\subsection{Blood glucose and insulin analysis}


Blood glucose was determined by a handheld glucometer validated for use in horses (OneTouch Ultra; LifeScan, Wayne, PA, USA) [42]. Hyperglycemia was diagnosed with blood glucose concentrations above $124 \mathrm{mg} / \mathrm{dL}$ [43]. Blood samples for insulin were collected in red top tubes and kept at room temperature to allow samples to clot for at least 1 hour. After this time, samples were kept in a cooler for transportation to the laboratory. All samples were centrifugated at $400 \mathrm{~g}$ for 10 minutes. Serum samples were harvested and transferred to new tubes and stored at $-20{ }^{\circ} \mathrm{C}$ until analysis. Insulin concentration was determined using a chemiluminescence assay (ADVIA Centaur, Siemens Healthcare Diagnostics) as previously described [44].

\subsection{Blood samples and neutrophil isolation}

Peripheral blood samples $(10 \mathrm{~mL})$ obtained by jugular venipuncture, at baseline and 120 min after oral glucose administration, were stored in sterile tubes containing $1 \mathrm{~mL}$ of $3.8 \% \mathrm{w} / \mathrm{v}$ trisodium citrate as anticoagulant. Blood samples for neutrophil isolation were kept at room temperature for 2 hours until further processing in the laboratory. A discontinuous gradient (Percoll®GE Healthcare) was carried out following the protocol described by Borlone et al., (2017). Briefly, $85 \%$ density Percoll $(4 \mathrm{~mL})$ were placed in the bottom of a $15 \mathrm{~mL}$ propylene tube, followed by $70 \%$ Percoll $(4 \mathrm{~mL})$ and blood on top $(4 \mathrm{~mL})$. Tubes were centrifugated for $45 \mathrm{~min}$ at $1500 \mathrm{xg}$ at room temperature for the obtention of two separately layers containing, mononuclear and PMNs cells respectively, for both times, baseline and $120 \mathrm{~min}$. PMNs layer was aspirated and neutrophils were washed with phosphate-buffered saline solution (PBS 1X). Cell pellet was resuspended in $2 \mathrm{~mL}$ of PBS $1 \mathrm{X}$, neutrophils were counted using a Neubauer chamber and immediately used.

\subsection{Apoptosis Assay}

In vitro neutrophil apoptosis was assessed at two-time points (baseline and $120 \mathrm{~min}$ ) using the FITC annexin $\mathrm{V}$ apoptosis detection kit (BD Biosciences) according to the manufacturer's protocol. Neutrophils $\left(1 \times 10^{6}\right)$ were resuspended in binding buffer $(100 \mu \mathrm{L})$ and transferred to cytometer tubes. To each tube, $5 \mu \mathrm{L}$ of FITC Annexin V (AV) and $5 \mu \mathrm{L}$ of Propidium iodide (PI) were added, and samples incubated in dark at $37^{\circ} \mathrm{C}$ for 15 minutes. After this time, samples were placed on ice after the addition of $200 \mu \mathrm{L}$ of binding buffer and immediately analyzed by flow cytometry (BD FACSCanto II cytometer, San Diego, CA, USA). Cells positive labeled for both, FITC AV and PI (AV+/PI+) were considered as apoptotic cells. Flow cytometry data was analyzed using FlowJo v10 software (FlowJo, LLC, USA).

\subsection{Phagocytosis Assay}

In vitro neutrophil phagocytic capacity was assessed at two-time points (baseline and $120 \mathrm{~min}$ ) using a commercial phagocytosis kit (pHrodo ${ }^{T M}$ Green E. Coli Bioparticles ${ }^{T M}$, ThermoFisher Scientific) according to the manufacturer's protocol. Briefly, $1 \times 10^{6}$ neutrophils were resuspended in PBS $(100 \mu \mathrm{L})$ and incubated in cytometry tubes with $25 \mu \mathrm{L}$ of pH-sensitive pHrodo ${ }^{\mathrm{Tm}}(2 \mathrm{mg} / \mathrm{mL})$ at $37^{\circ} \mathrm{C}$ for 30 minutes. Samples incubated at $4{ }^{\circ} \mathrm{C}$ for 30 minutes were used as negative controls. After incubation, engulfment of pHrodo bioparticles by cells were immediately analyzed by flow cytometry (BD FACSCanto II cytometer, San Diego, CA, USA). Flow cytometry data was analyzed using FlowJo v10 software (FlowJo, LLC, USA). 


\subsection{Respiratory burst production}

In vitro assessment of neutrophil ROS production at baseline and 120 min was determined using the luminol-dependent chemiluminescence method. In a white 96-well sterile plate, neutrophils resuspended in RPMI-1640 media (ThermoFisher Scientific) were aliquoted at a concentration of $3 \times 10^{5}$ neutrophils in $160 \mu \mathrm{L}$ per well. Next, luminol (20 $\mu \mathrm{L}$; Sigma Chemical CO.) was added to each well at a final concentration of $6.1 \mathrm{mM}$ and the plate was incubated in dark conditions for 30 minutes at $37^{\circ} \mathrm{C}$. After incubation, basal luminescence measurements were obtained, and ROS production was stimulated with the addition of $20 \mu \mathrm{L}$ of opsonized zymosan (OZ) (final concentration per well $0.1 \mathrm{mg} / \mathrm{mL}$ ). Chemiluminescence was measured with a luminometer (Varioskan®, ThermoFisher Scientific) for approximately 70 minutes. All the experiments were assessed in triplicate and results are expressed as relative light units (RLU). Area under the curve (AUC) values were obtained for each subject.

\subsection{Statistical Analysis}

Results are presented as least square mean (LSM) \pm standard error (SE). Normality of the data was assessed using the Kolmogorov-Smirnov test. AUC values were obtained for ROS production curves and used for further analysis. A repeated measures linear mixed model was fitted for the analysis of PMNs functions. Phagocytosis, apoptosis and AUC for ROS production were used as outcome variables, with time, group and the interaction between time and group as fixed effects, including subjects as a random effect. Tukey's HSD post hoc was performed when significant differences were found. Statistical analyses were performed with the commercial software RStudio (RStudio, USA). Graphs were obtained using GraphPad Prism 5.00 for windows (GraphPad Software, La Jolla California, USA). Statistical significance was defined at a $\mathrm{p}$-value $<0.05$.

\section{Results}

\subsection{Insulin and glucose response to in-feed OGT}

All animals tolerated the dextrose powder in the meal during the in-feed OGT and no adverse effects were observed in the horses. LSM \pm SE for blood glucose concentrations at baseline showed no significant differences $(P>0.05)$ between the lean insulin sensitive (lean-IS) $(92.1 \pm 13.9)$, obese insulin sensitive (obese-IS) $(91.6 \pm 16.4)$ and obese insulin resistance (obese-IR) $(111.4 \pm 16.4)$ groups (Fig. 1A). All baseline glucose measurements were within the reference range established for the species, and hyperglycemia was not detected. At $30 \mathrm{~min}$, a significant difference $(P<0.05)$ was observed between the lean-IS $(112.9 \pm 13.9)$ and obese-IR $(166.6 \pm 16.4)$ groups, but no significant differences were observed $(P$ $>0.05$ ) when the control group (lean-IS) was compared to the obese-IS animals (117.4 \pm 16.4$)$ (Fig. 1A). As showed in Fig. 1A, for the remaining times, 60, 90 and 120 minutes, no statistical significant differences $(P>0.05)$ were observed between the three groups (lean-IS, obese-IS and obese-IR). At 120 minutes, hyperglycemia was recorded in all three groups; lean-IS $(200.4 \pm 13.9)$, obese-IS $(201 \pm 16.4)$ and obese-IR (189.4 \pm 16.4$)$. 
Baseline serum insulin levels showed no significant differences $(P<0.05)$ between the lean-IS $(1.62 \pm$ 6.47), obese-IS (5.18 \pm 7.09$)$ and obese-IR (2.26 \pm 7.09$)$ groups (Fig. 1B). At $120 \mathrm{~min}$, a significant difference $(P<0.05)$ was observed in serum insulin concentration between the lean-IS $(17.78 \pm 6.47)$ and obese-IR (44.30 \pm 7.09$)$ groups, with no significant differences between the control (lean-IS, 17.78 \pm 6.47 ) and obese-IS group (30.92 \pm 7.09$)$ (Fig. 1B).

\subsection{Apoptosis}

At baseline, the percentage of live neutrophils showed no statistically significant differences $(P>0.05)$ between groups, lean-IS $(89.1 \pm 3.94)$, obese-IS $(93.4 \pm 3.94)$ and obese-IR $(90.7 \pm 3.94)$ (Fig. 2B).

Furthermore, no statistically significant differences $(P>0.05)$ were observed at 120 min between the three groups, lean-IS $(82.1 \pm 3.94)$, obese-IS $(93.8 \pm 3.94)$ and obese-IR $(87.7 \pm 3.94)$ (Fig. 2B). When comparing the percentage of apoptosis (cells positively labeled for FITC Annexin $V$ and propidium iodide) no significant differences $(P>0.05)$ were observed within or between groups at baseline (lean-IS 1.98 \pm 0.8 ; obese-IS $1.49 \pm 0.8$; obese-IR $1.69 \pm 0.8$ ) or 120 minutes (lean-IS $4.13 \pm 0.8$; obese-IS $2.17 \pm 0.8$; obese-IR $1.64 \pm 0.8)$ (Fig. 2C).

\subsection{Phagocytosis}

The total percentage of neutrophil phagocytic capacity at baseline (time 0 ) showed a statistically significant difference $(P<0.05)$ between the lean-IS $(59.2 \pm 3.09)$ and obese-IR $(35.6 \pm 3.78)$ groups (Fig. 3B). For the same time period, a significant difference $(P<0.05)$ was observed between the obese-IS $(60.8 \pm 3.38)$ and obese-IR (35.6 \pm 3.78$)$ groups (Fig. 3B). At $120 \mathrm{~min}$, no significant differences $(P>0.05)$ were observed between the three groups, lean-IS $(57.5 \pm 3.09)$, obese-IS $(58.1 \pm 3.38)$ and obese-IR $(49.2 \pm$ 3.78) (Fig. 3B). Within groups, neutrophil phagocytic capacity of the obese-IR group showed a significant difference $(P=0.007)$ between baseline $(35.6 \pm 3.78)$ and $120 \mathrm{~min}(49.2 \pm 3.78)$ (Fig. 3B). No significant differences $(P>0.05)$ were observed between the two time points for the lean-IS $(59.2 \pm 3.09 ; 57.5 \pm 3.09)$ and obese-IS groups $(60.8 \pm 3.38 ; 58.1 \pm 3.38)$ (Fig. 3B).

\subsection{Reactive oxygen species}

AUC for ROS production at baseline (time 0$)$ was statistically significantly different $(P=0.0001)$ for the obese-IS group ( $3743 \pm 236)$ when compared to both, the lean-IS $(1196 \pm 215)$ and obese-IR $(1423 \pm 236)$ groups (Fig. 4C). For the 120 min time point, a significant difference $(P<0.0001)$ was observed between the obese-IS group (3094 \pm 236$)$ when compared to the lean-IS $(741 \pm 215)$, and obese-IR $(1039 \pm 236)$ groups (Fig. 4C). Within groups, the AUC for ROS production of the lean-IS group showed a statistically significant difference $(P<0.05)$ between baseline $(1196 \pm 215)$ and $120 \mathrm{~min}(741 \pm 215)$ (Fig. 4C). A statistically significant difference $(P<0.05)$ was also observed for the obese-IS group between the two time points ( $3743 \pm 236 ; 3094 \pm 236)$ (Fig. 4 C). The obese-IR group showed no significant difference $(\mathrm{P}>$ 0.05 ) between baseline (1423 \pm 236$)$ and $120 \mathrm{~min}(1039 \pm 236)$ (Fig. 4C).

\section{Discussion}


The present study aimed to evaluate changes in peripheral blood neutrophil apoptosis, phagocytosis and ROS production, in response to acute hyperglycemia, in horses with obesity and insulin resistance. Our results provide evidence that obesity per se can modulate neutrophil oxidative burst independently of insulin sensitivity. Moreover, obesity in association to IR impairs peripheral blood neutrophil phagocytosis but not ROS production. Induced hyperglycemia increased phagocytosis in the obese-IR group and diminished ROS production in all study groups, although this decrease was not significant for the obeseIR group. To the authors' knowledge this is the first report regarding the effect of in vivo hyperglycemia, obesity and IR, on these aspects of neutrophil functionality.

The respiratory burst is an important oxygen-dependent function of neutrophils, highly involved in their bactericidal capacity [46]. In the present study, a marked increase in basal ROS production was observed in the obese-IS group when compared to both, the lean-IS and obese-IR groups. These results are consistent with those reported by Brotfain et al. (2015), who reported overproduction of ROS in morbidly obese non-diabetic human patients. Conversely, obese-IR horses showed a minimal increase in ROS production, with results similar to those observed in the lean-IS group. This was an interesting and unexpected result. In humans, IR is a condition characterized by high ROS production and is a key feature of type 2 diabetes mellitus [47]. Studies conducted in subjects with type 2 diabetes have widely documented this marked increase in ROS production [48, 49]. Furthermore, high levels of ROS have been linked to endothelial damage in humans suffering from metabolic disorders [50]. Likewise, endothelial dysfunction associated with endocrinophatic laminitis has been reported in horses with metabolic disturbances such as metabolic syndrome [51]. In horses few studies have explored the relation between metabolic disorders and neutrophil respiratory burst $[13,14]$. Holbrook et al., (2012) observed a marked increase in ROS production in hyperinsulinemic obese horses when compared to a lean group. Although none of the animals tested in our study were hyperinsulinemic, the contradictory results obtained between Holbrook's and the present study requires further research. On the other hand, in our study acute hyperglycemia induced a significant reduction of neutrophil ROS production in both insulin sensitive groups, but no significant decrease was observed in the obese-IR group. In healthy humans, induced acute hyperglycemia assessed through different clamp techniques failed to influence neutrophils oxidative burst response [52,53]. However, in diabetic patients, hyperglycemia is described as an important priming factor of neutrophils responsible for their excessive response under stimulation, including an exaggerated production of ROS $[54,55]$. Furthermore, hyperglycemia stimulates the activation of the polyol pathway, an alternative metabolic route, in which glucose at high concentrations is converted to sorbitol by the enzyme aldose reductase [31]. In diabetic patients the activation of this metabolic pathway has been linked with increased neutrophils ROS production, which levels were restored after the use of an aldose reductase inhibitor [56]. Although pancreatic exhaustion and consequent poor glycemic control are uncommon in horses [19], cases of type 2 diabetes have been reported [57] and an activation of this alternative pathway cannot be discarded. To date, no data is available regarding the effect of hyperglycemia and alternative metabolic routes activation on neutrophil functions of horses with metabolic disorders. Thus, these results suggest that metabolic status of horses 
and hyperglycemia affect the responsiveness of blood neutrophils, with an exacerbated oxidative burst response observed in the obese-IS group, which could play a detrimental role in horses' health.

Regarding phagocytosis, the obese-IR group showed an important decrease in the phagocytic rate of neutrophils compared to the insulin-sensitive groups, regardless of their body condition score. Consistent with our results, a decreased PMNs phagocytic activity has been reported in humans and rats with type 2 diabetes [58-60]. Furthermore, results from the insulin sensitive groups (lean-IS and obese-IS) are consistent with those described by Brotfain et al., (2015), where no differences in neutrophil phagocytosis were found between lean and non-diabetic morbidly obese subjects. Few studies in horses have aimed to determinate the effect of metabolic diseases on neutrophil phagocytosis $[13,14]$. In a study conducted in obese-hyperinsulinemic horses, no differences were reported in the phagocytosis of bacteria when compared to a lean-control group [13]. Although our results are not consistent with that previous report, our study population differed in that none of the animals were hyperinsulinemic, but rather, insulin resistant, which is a more prevalent condition in clinical patient populations. Interestingly, hyperglycemia in the obese-IR group appears to increase the phagocytic rate of neutrophils. This finding differs from available in vitro data, in which hyperglycemia has been reported to impair and decrease phagocytosis of human PMNs $[33,61]$. On the other hand, apoptosis is an important neutrophil function for the maintenance of tissue homeostasis [62]. Alterations in the process of neutrophil programmed cell death has been associated with chronic inflammation, tissue injury and some diseases in humans [63]. In the present study, apoptotic capacity of neutrophils appears to not be influenced by either, hyperglycemia or the metabolic status of horses. Although a slightly decrease in the apoptotic rate was observed in the two obese groups, no significative differences were reached. Thus far, little information is known regarding the relation between metabolic disorders and neutrophil apoptosis in horses. Only one study reported an increased expression of apoptotic related proteins in adipose and hepatic tissue of horses with equine metabolic syndrome [64]. However, other reports have studied the effects of different diseases, such as asthma [65], colitis and lipopolysaccharide infusion [66, 67], upon neutrophil apoptosis. Interestingly, delayed neutrophil apoptotic rates have been reported in the studies mentioned above. Diverse studies in humans and mice had demonstrated that both, a dysfunctional metabolic status like type 2 diabetes mellitus and hyperglycemia are able to decrease the rate of neutrophil apoptosis $[33,68,69]$. Our study do not support similar findings in the equine species, but it must be acknowledged that only spontaneous neutrophil apoptosis was assessed, in absence of stimuli reagents. Stimuli are able to enhance the prolonged lifespan of neutrophils, and therefore differences are easier to observe when metabolic disturbances are present [69].

The low number of horses tested per group is the main limitation of the present study. A larger study population will be able to increase the power of the test and provide more robust evidence. Furthermore, the effect of hyperglycemia over neutrophils functions was determined at a single time point after the carbohydrate overdose. In vitro experiments with different glucose concentrations, and in vivo trials with persistent hyperglycemia, could provide more information on the effect of glucose on neutrophil functions. Additionally, only one laboratory technique was performed for the assessment of each neutrophil function in this study. Future experimental studies testing different techniques and stimuli, and 
including other neutrophil functions such as chemotaxis, adherence capacity and NETosis should be explored. Moreover, future studies should include insulin dysregulated animals, as this group was not included in the present report.

\section{Conclusion}

In conclusion, our results address the effect of obesity as an isolated factor and in association with IR on blood neutrophil functionality in adult horses, indicating that phagocytosis and oxidative burst functions can be altered by metabolic status itself and by acute hyperglycemia in horses. Furthermore, our results support the fact that endocrinology is a dynamic science, and that the extrapolation of results from other species to the equine population should be done cautiously.

\section{Abbreviations}

AUC: area under the curve

$\mathrm{AV}$ : annexin $\mathrm{V}(\mathrm{AV})$

BCS: body condition score

ID: insulin dysregulation

IL-1: interleukin 1

IR: insulin resistance

IS: insulin sensitive

ITT: insulin tolerance test

LSM: least square mean

NETs: neutrophils extracellular traps

OGT: oral glucose test

OZ: opsonized zymosan

PBS: phosphate-buffered saline solution

PI: propidium iodide

PMNs: polymorphonuclear cells

RLU: relative light units 
ROS: reactive oxygen species

SE: standard error

TNFa : tumor necrosis factor alpha

\section{Declarations}

\section{Ethics approval and consent to participate}

This study was approved by the Bioethics Committee for the Use of Animals in Research of Austral University of Chile (Resolution $\left.N^{\circ} 308 / 2018\right)$. An informed consent was signed before the performance of the study for privately owned horses.

\section{Consent for publication}

Not applicable.

\section{Competing interests}

The authors declare no conflict of interest with respect to the research, authorship, and/or publication of this article.

\section{Funding}

This work was supported by Universidad Austral de Chile funding nº DID S-2018-24. The author confirm that the funders had no role in the study design, data collection and analysis, decision to publish, preparation of the manuscript or selection of this journal.

\section{Author's contributions}

CS and BU conceived the study, participated in the design of the study, sampling and processing and were primarily responsible for manuscript writing. GM, CH and RB participated in the design of the study and assisted in data interpretation and manuscript revisions. GE, NM, CH and GG participated in laboratory technique settings. All authors have read and approved the manuscript.

\section{Acknowledgements}

Not applicable. 


\section{Availability of data and materials}

All data is available upon request.

\section{References}

1. Chandler M, Cunningham S, Lund EM, Khanna C, Naramore R, Patel A, et al. Obesity and Associated Comorbidities in People and Companion Animals: A One Health Perspective. J Comp Pathol. 2017;156:296-309.

2. Zheng Y, Ley SH, Hu FB. Global aetiology and epidemiology of type 2 diabetes mellitus and its complications. Nat Rev Endocrinol. 2018;14:88-98.

3. Holst BS, Gustavsson MH. Animal obesity: causes, consequences and comparative aspects. Acta Vet Scand. 2016;58 (Suppl:56.

4. Thatcher CD, Pleasant RS, Geor RJ, Elvinger F. Prevalence of Overconditioning in Mature Horses in Southwest Virginia during the Summer. 2012.

5. Frank N, Tadros EM. Insulin dysregulation. Equine Vet J. 2014;46:103-12.

6. Asplin KE, Sillence MN, Pollitt CC, McGowan CM. Induction of laminitis by prolonged hyperinsulinaemia in clinically normal ponies. Vet J. 2007;174:530-5.

7. de Laat MA, McGowan CM, Sillence MN, Pollitt CC. Equine laminitis: Induced by $48 \mathrm{~h}$ hyperinsulinaemia in Standardbred horses. Equine Vet J. 2010;42:129-35. doi:10.2746/042516409X475779.

8. de Laat MA, Sillence MN, McGowan CM, Pollitt CC. Continuous intravenous infusion of glucose induces endogenous hyperinsulinaemia and lamellar histopathology in Standardbred horses. Vet J. 2012;191:317-22. doi:10.1016/j.tvjl.2011.07.007.

9. Patterson-Kane JC, Karikoski NP, McGowan CM. Paradigm shifts in understanding equine laminitis. Veterinary Journal. 2018;231:33-40. doi:10.1016/j.tvjl.2017.11.011.

10. Patel PS, Buras ED, Balasubramanyam A. The role of the immune system in obesity and insulin resistance. J Obes. 2013;2013.

11. Daryabor G, Kabelitz D, Kalantar K. An update on immune dysregulation in obesity-related insulin resistance. Scand J Immunol. 2019;89:e12747.

12. Elzinga S, Reedy S, Barker VD, Chambers TM, Adams AA. Humoral and cell-mediated immune responses to influenza vaccination in equine metabolic syndrome (EMS) horses. Vet Immunol Immunopathol. 2018;199 March:32-8.

13. Holbrook TC, Tipton T, McFarlane D. Neutrophil and cytokine dysregulation in hyperinsulinemic obese horses. Vet Immunol Immunopathol. 2012;145:283-9.

14. McFarlane D, Hill K, Anton J. Neutrophil function in healthy aged horses and horses with pituitary dysfunction. Vet Immunol Immunopathol. 2015;165:99-106. 
15. Miller AB, Loynachan AT, Bush HM, Hart K, Barker VD, Campana-Emard AG, et al. Effects of pituitary pars intermedia dysfunction and Prascend (pergolide tablets) treatment on endocrine and immune function in horses. Domest Anim Endocrinol. 2021;74:106531.

16. Carter RA, Treiber KH, Geor RJ, Douglass L, Harris PA. Prediction of incipient pasture-associated laminitis from hyperinsulinaemia, hyperleptinaemia and generalised and localised obesity in a cohort of ponies. Equine Vet J. 2009;41:171-8.

17. Coleman MC, Whitfield-Cargile CM, Madrigal RG, Cohen ND. Comparison of the microbiome, metabolome, and lipidome of obese and non-obese horses. PLoS One. 2019;14:e0215918. doi:10.1371/journal.pone.0215918.

18. Biddle AS, Tomb JF, Fan Z. Microbiome and blood analyte differences point to community and metabolic signatures in lean and obese horses. Front Vet Sci. 2018;5 SEP:225. doi:10.3389/fvets.2018.00225.

19. Morgan R, Keen J, McGowan C. Equine metabolic syndrome. Vet Rec. 2015;177(7):173-9.

20. Brotfain E, Hadad N, Shapira Y, Avinoah E, Zlotnik A, Raichel L, et al. Neutrophil functions in morbidly obese subjects. Clin Exp Immunol. 2015;181:156-63.

21. De Heredia FP, Gómez-Martínez S, Marcos A. Obesity, inflammation and the immune system. Proc Nutr Soc. 2012;71:332-8.

22. Vieira-Potter VJ. Inflammation and macrophage modulation in adipose tissues. Cell Microbiol. 2014;16:1484-92.

23. Vick MM, Adams AA, Murphy BA, Sessions DR, Horohov DW, Cook RF, et al. Relationships among inflammatory cytokines, obesity, and insulin sensitivity in the horse. J Anim Sci. 2007;85:1144-55.

24. Adams AA, Katepalli MP, Kohler K, Reedy SE, Stilz JP, Vick MM, et al. Effect of body condition, body weight and adiposity on inflammatory cytokine responses in old horses. Vet Immunol Immunopathol. 2009;127:286-94.

25. Suagee JK, Corl BA, Crisman M V., Pleasant RS, Thatcher CD, Geor RJ. Relationships between Body Condition Score and Plasma Inflammatory Cytokines, Insulin, and Lipids in a Mixed Population of Light-Breed Horses. J Vet Intern Med. 2013;27:157-63.

26. Selim S, Elo K, Jaakkola S, Karikoski N, Boston R, Reilas T, et al. Relationships among body condition, insulin resistance and subcutaneous adipose tissue gene expression during the grazing season in mares. PLoS One. 2015;10:1-20.

27. Burns TA, Geor RJ, Mudge MC, McCutcheon LJ, Hinchcliff KW, Belknap JK. Proinflammatory Cytokine and Chemokine Gene Expression Profiles in Subcutaneous and Visceral Adipose Tissue Depots of Insulin-Resistant and Insulin-Sensitive Light Breed Horses. J Vet Intern Med. 2010;24:9329.

28. Wray H, Elliott J, Bailey SR, Harris PA, Menzies-Gow NJ. Plasma concentrations of inflammatory markers in previously laminitic ponies. Equine Vet J. 2013;45:546-51.

29. Rosales C. Neutrophil: A cell with many roles in inflammation or several cell types? Front Physiol. 2018;9 FEB:113. 
30. Anderson SL, Singh B. Equine neutrophils and their role in ischemia reperfusion injury and lung inflammation. Cell and Tissue Research. 2018;371:639-48.

31. Alba-Loureiro TC, Munhoz CD, Martins JO, Cerchiaro GA, Scavone C, Curi R, et al. Neutrophil function and metabolism in individuals with diabetes mellitus. Brazilian J Med Biol Res. 2007;40:1037-44.

32. Menegazzo L, Ciciliot S, Poncina N, Mazzucato M, Persano M, Bonora B, et al. NETosis is induced by high glucose and associated with type 2 diabetes. Acta Diabetol. 2015;52:497-503.

33. Jafar $\mathrm{N}$, Edriss $\mathrm{H}$, Nugent $\mathrm{K}$. The effect of short-term hyperglycemia on the innate immune system. Am J Med Sci. 2016;351:201-11.

34. Fedorova N V., Ksenofontov AL, Serebryakova M V., Stadnichuk VI, Gaponova T V., Baratova LA, et al. Neutrophils release metalloproteinases during adhesion in the presence of insulin, but cathepsin $G$ in the presence of glucagon. Mediators Inflamm. 2018;2018.

35. Wierusz-Wysocka B, Wysocki H, Wykrętowicz A, Klimas R. The influence of increasing glucose concentrations on selected functions of polymorphonuclear neutrophils. Acta Diabetol Lat. 1988;25:283-8.

36. Ortmeyer J, Mohsenin V. Inhibition of phospholipase D and superoxide generation by glucose in diabetic neutrophils. Life Sci. 1996;59:255-62.

37. Wang L, Zhou X, Yin Y, Mai Y, Wang D, Zhang X. Hyperglycemia Induces Neutrophil Extracellular Traps Formation Through an NADPH Oxidase-Dependent Pathway in Diabetic Retinopathy. Front Immunol. 2019;9.

38. Salinas C, Espinosa G, Morales N, Henríquez C, Morán G, Gajardo G, et al. Assessment of peripheral blood neutrophil respiratory burst, phagocytosis and apoptosis in obese non-insulin dysregulated horses. Res Vet Sci. 2020. doi:10.1016/j.rvsc.2020.06.013.

39. Henneke DR, Potter GD, Kreider JL, Yeates BF. Relationship between condition score, physical measurements and body fat percentage in mares. Equine Vet J. 1983;15:371-2.

40. Bertin FR, Sojka-Kritchevsky JE. Comparison of a 2-step insulin-response test to conventional insulinsensitivity testing in horses. Domest Anim Endocrinol. 2013;44:19-25.

41. Meier A, Reiche D, de Laat M, Pollitt C, Walsh D, Sillence M. The sodium-glucose co-transporter 2 inhibitor velagliflozin reduces hyperinsulinemia and prevents laminitis in insulin-dysregulated ponies. PLoS One. 2018;13:1-13.

42. Slough TL, Gunkel CD, Murray LW, Drouillard JS. A comparison of methodologies for measuring glucose concentrations in the horse. Prof Anim Sci. 2011;27:204-14.

43. Bertin F, Ruffin-Taylor D, Stewart AJ. Insulin dysregulation in horses with systemic inflammatory response syndrome. 2018.

44. Meier AD, de Laat MA, Reiche DB, Pollitt CC, Walsh DM, McGree JM, et al. The oral glucose test predicts laminitis risk in ponies fed a diet high in nonstructural carbohydrates. Domest Anim Endocrinol. 2018;63:1-9. doi:10.1016/j.domaniend.2017.10.008. 
45. Borlone C, Morales N, Henriquez C, Folch H, Olave C, Sarmiento J, et al. In Vitro effects of tamoxifen on equine neutrophils. Res Vet Sci. 2017;110.

46. Kobayashi SD, Malachowa N, DeLeo FR. Neutrophils and Bacterial Immune Evasion. J Innate Immun. 2018;10:432-41.

47. Houstis N, Rosen ED, Lander ES. Reactive oxygen species have a causal role in multiple forms of insulin resistance. Nature. 2006;440:944-8.

48. Kaneto $\mathrm{H}$, Katakami N, Matsuhisa M, Matsuoka TA. Role of reactive oxygen species in the progression of type 2 diabetes and atherosclerosis. Mediators Inflamm. 2010;2010.

49. Fakhruddin S, Alanazi W, Jackson KE. Diabetes-Induced Reactive Oxygen Species: Mechanism of Their Generation and Role in Renal Injury. J Diabetes Res. 2017;2017:8379327.

50. Incalza MA, D'Oria R, Natalicchio A, Perrini S, Laviola L, Giorgino F. Oxidative stress and reactive oxygen species in endothelial dysfunction associated with cardiovascular and metabolic diseases. Vascular Pharmacology. 2018;100:1-19.

51. Morgan RA, Keen JA, Walker BR, Hadoke PWF. Vascular Dysfunction in Horses with Endocrinopathic Laminitis. 2016.

52. Fejfarová V, Jirkovská A, Lupínková J, Kovář J, Kalanin J, Stříž I, et al. Effect of acute hyperglycemia and/or hyperinsulinemia on polymorphonuclear functions in healthy subjects. Metabolism. 2006;55:811-8.

53. Stegenga ME, Crabben SNV Der, Dessing MC, Pater JM, Van Den Pangaart PS, De Vos AF, et al. Effect of acute hyperglycaemia and/or hyperinsulinaemia on proinflammatory gene expression, cytokine production and neutrophil function in humans. Diabet Med. 2008;25:157-64.

54. Omori K, Ohira T, Uchida Y, Ayilavarapu S, Batista EL, Yagi M, et al. Priming of neutrophil oxidative burst in diabetes requires preassembly of the NADPH oxidase. J Leukoc Biol. 2008;84:292-301.

55. Karima M, Kantarci A, Ohira T, Hasturk H, Jones VL, Nam B-H, et al. Enhanced superoxide release and elevated protein kinase $\mathrm{C}$ activity in neutrophils from diabetic patients: association with periodontitis. J Leukoc Biol. 2005;78:862-70.

56. Joshi MB, Ahamed R, Hegde M, Nair AS, Ramachandra L, Satyamoorthy K. Glucose induces metabolic reprogramming in neutrophils during type 2 diabetes to form constitutive extracellular traps and decreased responsiveness to lipopolysaccharides. Biochim Biophys Acta - Mol Basis Dis. 2020;1866:165940.

57. Johnson PJ, Scotty NC, Wiedmeyer C, Messer NT, Kreeger JM. Diabetes mellitus in a domesticated Spanish Mustang. J Am Vet Med Assoc. 2005;226:584-8.

58. Alba-Loureiro TC, Hirabara SM, Mendonca $\$ JR, Curi R, Pithon-Curi TC. Diabetes causes marked changes in function and metabolism of rat neutrophils. J Endocrinol. 2006;188:295-303.

59. Panneerselvam S, Govindasamy S. Sodium molybdate improves the phagocytic function in alloxaninduced diabetic rats. Chem Biol Interact. 2003;145:159-63. 
60. Alba-Loureiro TC, Munhoz CD, Martins JO, Cerchiaro GA, Scavone C, Curi R, et al. Neutrophil function and metabolism in individuals with diabetes mellitus. Brazilian J Med Biol Res. 2007;40:1037-44. doi:10.1590/S0100-879X2006005000143.

61. Schuetz P, Castro P, Shapiro NI. Diabetes and sepsis: Preclinical findings and clinical relevance. Diabetes Care. 2011;34:771-8.

62. Greenlee-Wacker MC. Clearance of apoptotic neutrophils and resolution of inflammation. Immunol Rev. 2016;273:357-70.

63. McCracken JM, Allen LAH. Regulation of human neutrophil apoptosis and lifespan in health and disease. J Cell Death. 2014.

64. Marycz K, Kornicka K, Basinska K, Czyrek A. Equine Metabolic Syndrome Affects Viability, Senescence, and Stress Factors of Equine Adipose-Derived Mesenchymal Stromal Stem Cells: New Insight into EqASCs Isolated from EMS Horses in the Context of Their Aging. Int J Mol Sci. 2018;19:165.

65. Niedzwiedz A, Jaworski Z, Tykalowski B, Smialek M. Neutrophil and macrophage apoptosis in bronchoalveolar lavage fluid from healthy horses and horses with recurrent airway obstruction (RAO). BMC Vet Res. 2014;10.

66. Anderson SL, Singh B. Neutrophil apoptosis is delayed in an equine model of colitis: Implications for the development of systemic inflammatory response syndrome. Equine Vet J. 2017;49:383-8.

67. Anderson SL, Townsend HGG, Singh B. Role of toll-like receptor 4 and caspase-3,-8, and-9 in lipopolysaccharide-induced delay of apoptosis in equine neutrophils. Am J Vet Res. 2018;79:424-32.

68. Manosudprasit A, Kantarci A, Hasturk H, Stephens D, Van Dyke TE. Spontaneous PMN apoptosis in type 2 diabetes and the impact of periodontitis. J Leukoc Biol. 2017;102:1431-40.

69. Hanses F, Park S, Rich J, Lee JC. Reduced neutrophil apoptosis in diabetic mice during staphylococcal infection leads to prolonged Tnfa production and reduced neutrophil clearance. PLoS One. 2011;6:e23633.

\section{Figures}


A

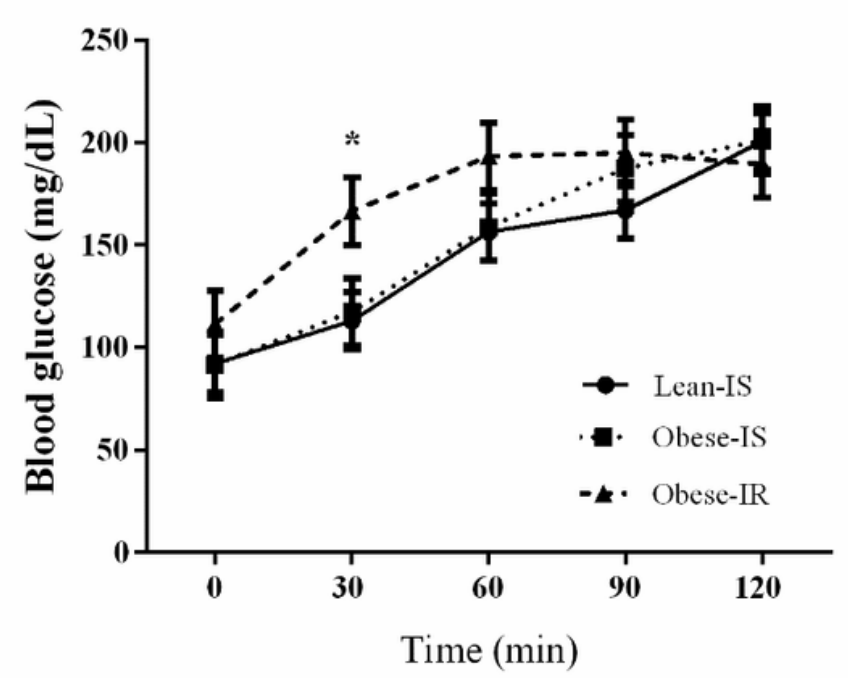

B

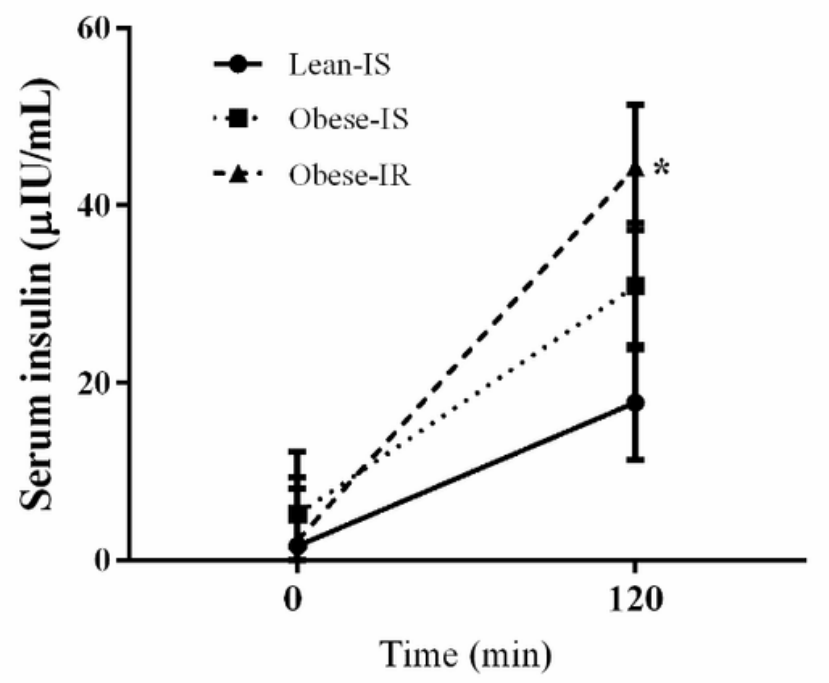

Figure 1

Least square mean \pm standard error of $(A)$ blood glucose and $(B)$ serum insulin concentrations measured between groups during an infeed-OGT. * Statistically significant differences compared to Lean-IS group for that time point $(P<0.05)$. Lean-IS $n=7$; obese-IS $n=5$ and obese-IR $n=5$; IS, insulin sensitive; IR, insulin resistance; OGT, oral glucose test. 
A

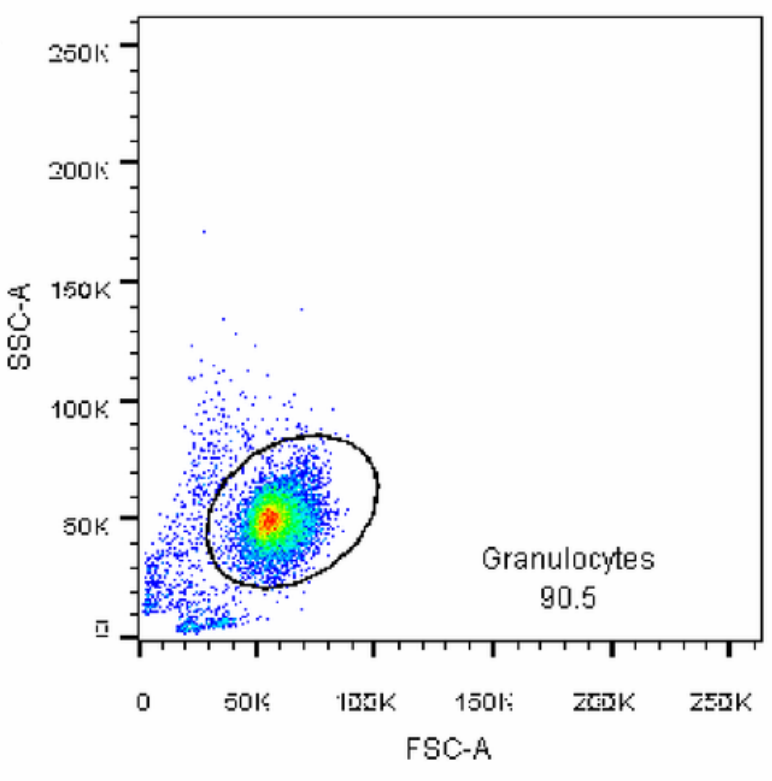

B

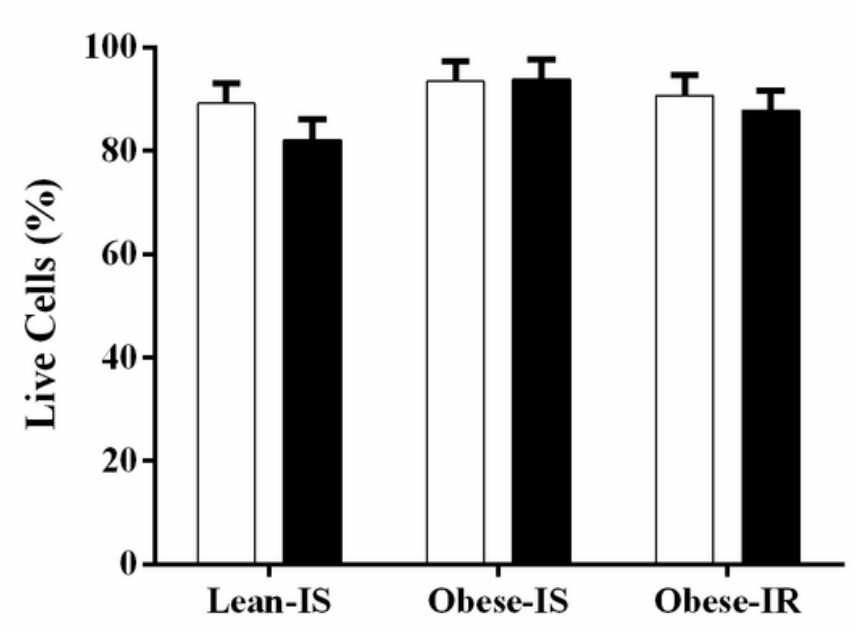

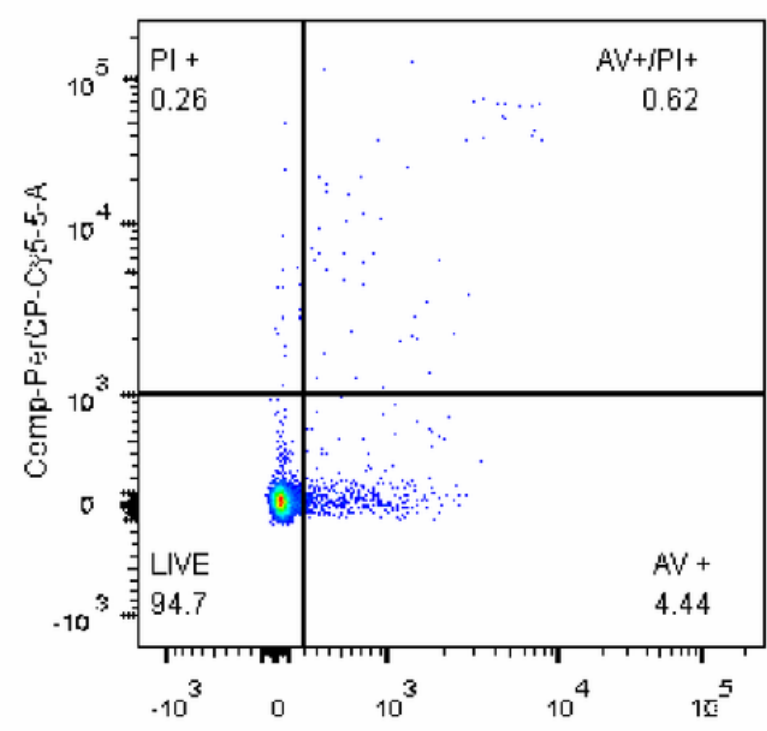

C

Crmp-FITC-M

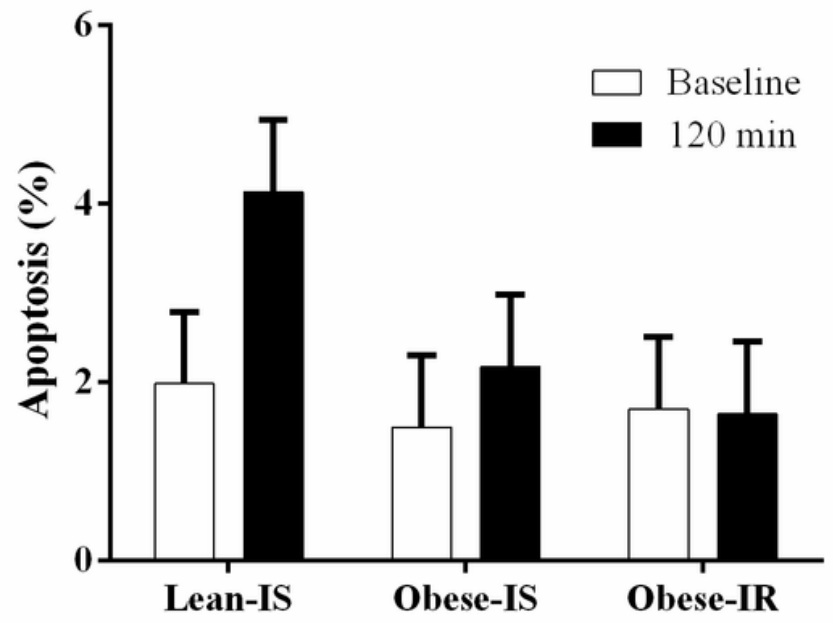

Figure 2

Purified peripheral blood neutrophils labeled with annexin V-FITC (AV) and Propidium lodide (PI) measured by flow cytometry among groups. (A) Flow cytometry gating strategy for cell viability selection. (B) Percentage of live and (C) apoptotic neutrophils (sum of cells AV+ and AV+/IP+) from the three study groups observed at baseline (time 0 ) and 120 min during an infeed-OGT challenge. No statistically significant differences were observed among groups $(P>0.05)$. Data is presented as least square mean \pm standard error. Lean-IS $n=7$; obese-IS $n=5$ and obese-IR $n=5$; ${ }^{\star} p<0.05$; IS, insulin sensitive; IR, insulin resistance; OGT, oral glucose test. 

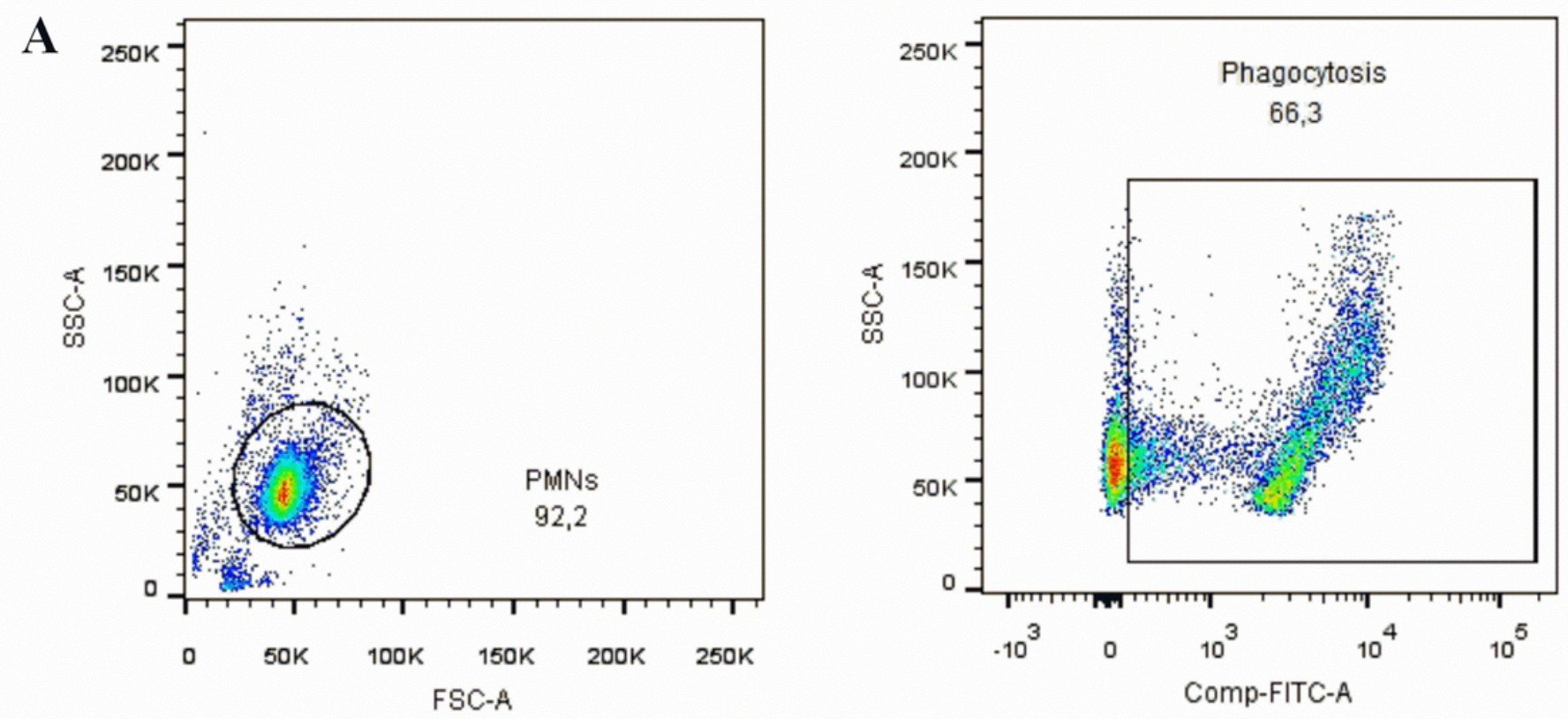

B

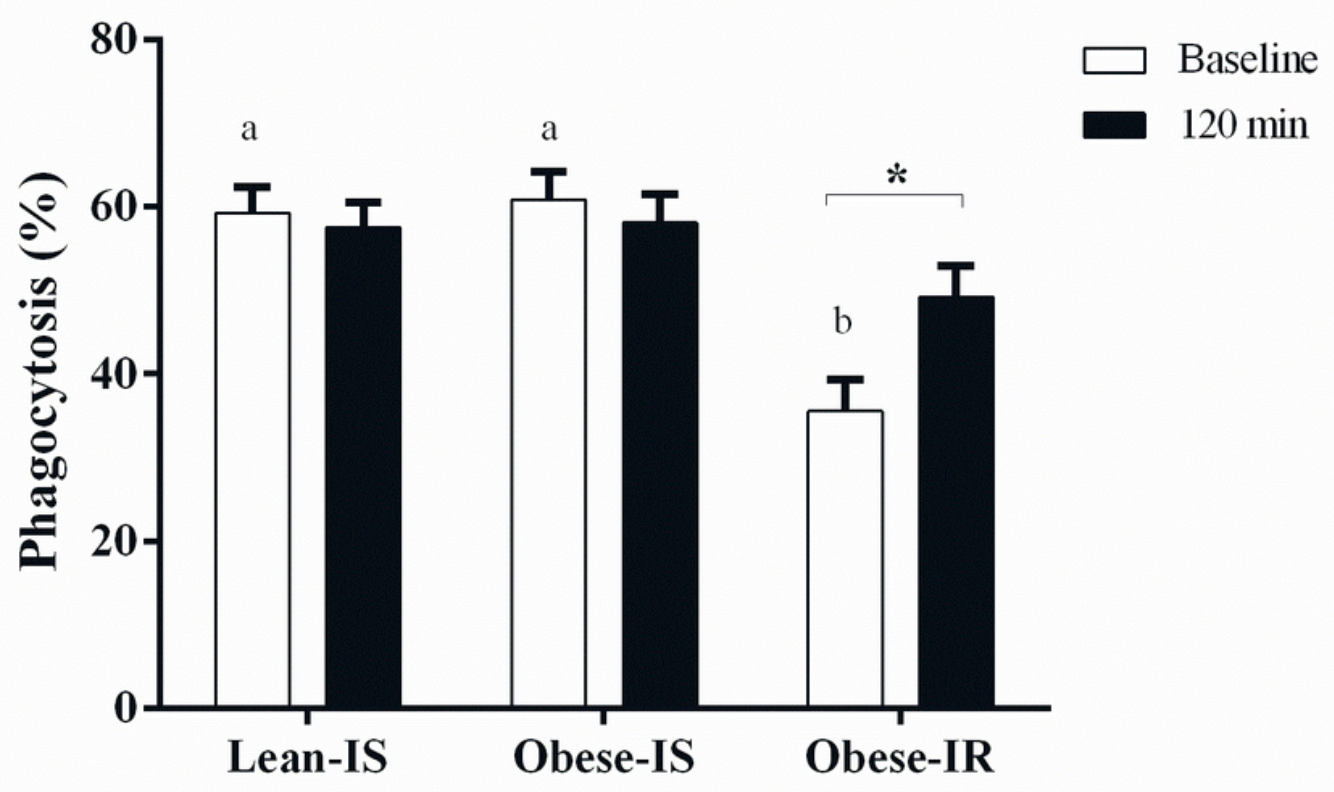

Figure 3

Equine peripheral blood neutrophil phagocytic capacity assessed through a pH-dependent dye measured by flow cytometry during an infeed-OGT challenge. (A) Representation of neutrophil and bioparticles uptake gating strategy. (B) Percentage of neutrophil phagocytic activity at baseline (time 0 ) and 120 min for the three study groups. * Statistically significant difference for the two-time points within a group $(P<0.05)$. Means for the same time point between groups with different superscripts differ significantly $(P<0.05)$. Data is presented as least square mean \pm standard error. Lean-IS $n=7$; obese-IS $n=5$ and obeseIR $n=5$; * $\mathrm{p}<0.05$; IS, insulin sensitive; IR, insulin resistance; OGT, oral glucose test. 
A

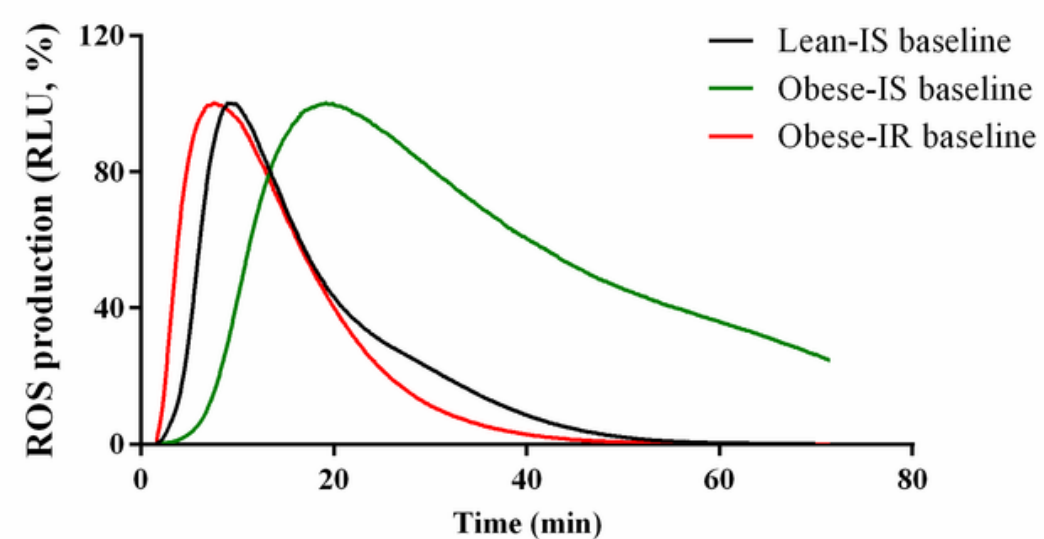

B
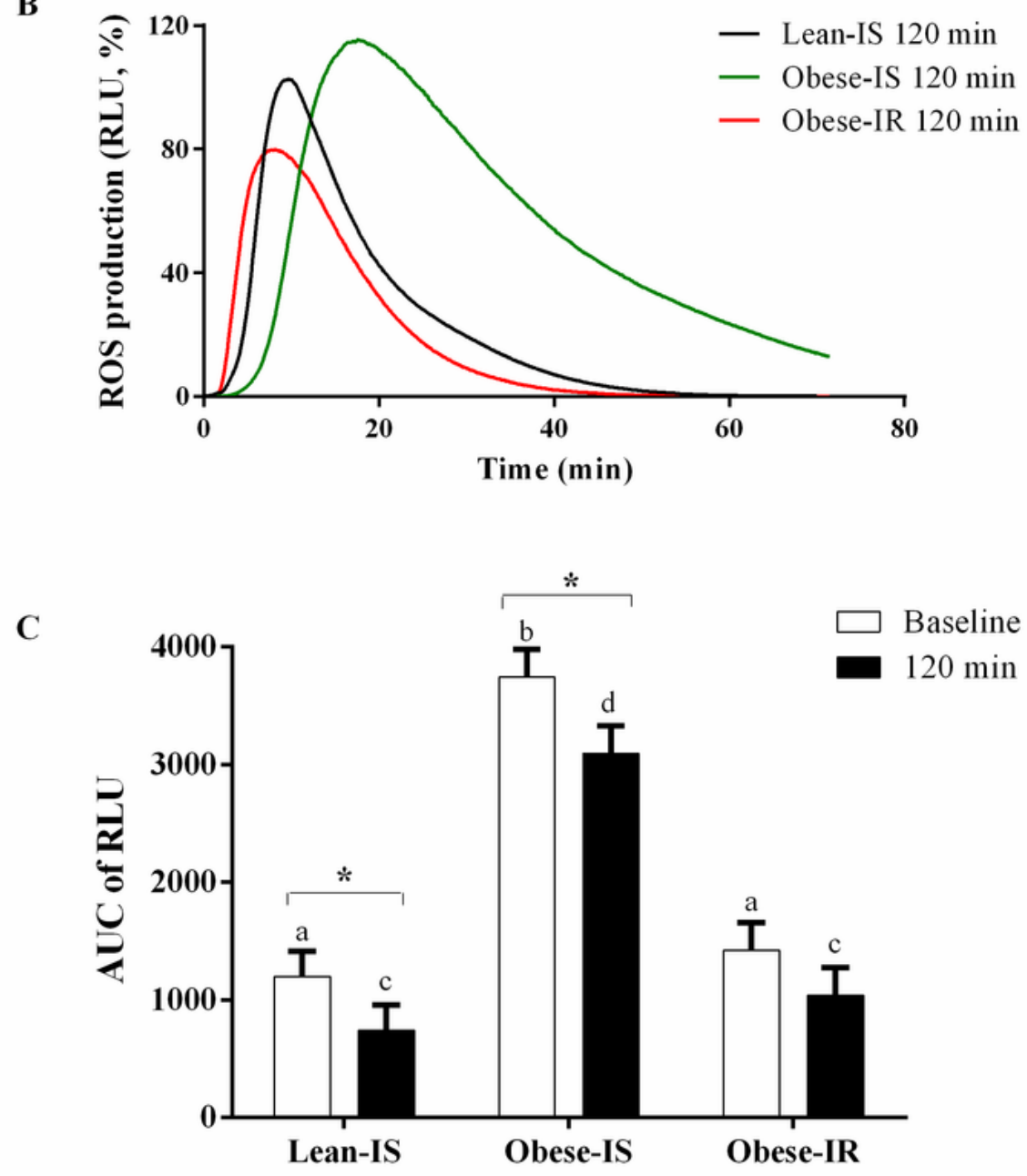

Figure 4

Neutrophils ROS production in response to the physiological agonist opsonized zymosan (OZ) measured by chemiluminescence among groups during an infeed-OGT. $(A, B)$ Representative chemiluminescence curves of the three groups for baseline (time 0 ) and 120 minutes through time. * Statistically significant difference for the two-time points within a group $(P<0.05)$. Means for the same time point between groups with different superscripts letters differs significantly $(P<0.05)$. Data is presented as least square 
mean \pm standard error. Lean-IS $n=7$; obese-IS $n=5$ and obese-IR $n=5 ;{ }^{*} p<0.05 ; R O S$, reactive oxygen species; AUC, area under the curve; RLU, relative light units; IS, insulin sensitive; IR, insulin resistance; OGT, oral glucose test.

\section{Supplementary Files}

This is a list of supplementary files associated with this preprint. Click to download.

- SalinasARRRIVE2.0checklist.xlsx 